\title{
Specimen Temperature Rises in a Nanosecond Laser Pulsed 3-D Atom Probe
}

\author{
A. Cerezo, ${ }^{*}$ P.H. Clifton, ${ }^{* *}$ and G.D.W. Smith* \\ * Department of Materials, University of Oxford, Parks Road, Oxford OX1 3PH, UK \\ ** Oxford nanoScience, 4-6 Carters Lane, Kiln Farm, Milton Keynes, MK11 3ER, UK
}

The 3-dimensional atom probe (3DAP) is capable of producing three-dimensional chemical maps at the atomic scale in a volume typically $20 \mathrm{~nm}$ x $20 \mathrm{~nm} \times 100 \mathrm{~nm}$ [1]. Most 3DAP instruments use a high voltage pulse to generate pulsed field evaporation, limiting the instrument to analysis of high conductivity materials (metals and highly-doped semiconductors). Laser pulsing has been used to extend the applicability of the technique to semiconductors allowing, for example, the analysis of multiple quantum well structures [2]. A recent paper has suggested that use of nanosecond laser pulses in this application produces very high temperature rises, producing a high degree of surface diffusion which will destroy the technique's spatial resolution [3]. However, previous measurements have indicated peak temperature rises from the base temperature of $50-80 \mathrm{~K}$ to approximately room temperature under typical laser pulsing conditions [4]. This would not be sufficient to generate significant surface diffusion over the limited duration of the temperature rise. A pulsed laser atom probe analysis of ordered Ni3Al is shown in Fig. 1, obtained using 5ns pulses from a Nd-YAG laser. The sequence of atom detection is characteristic of the L12 ordered structure, which would not be observed if there was a significant degradation in lateral spatial resolution.

Although it is not possible to directly measure peak specimen temperature (except under very high laser pulse energies when surface diffusion does occur), charge states of field evaporated ions can be used to give some indication of the temperature rise [5]. Mass spectra have been obtained from specimens of tungsten and intrinsic silicon using $0.6 \mathrm{~ns}$ laser pulses from a Nd-YAG laser operating at $35 \mathrm{kHz}$. In the mass spectrum from tungsten, the ratio of $\mathrm{W} 3+$ ions to $\mathrm{W} 4+$ ions detected using laser pulsing at standard conditions was a factor of 20 higher than that observed during the voltage pulsing (620 and 31 respectively). However, no doubly charged ions were observed. Measurements of charge state variations show that $2+$ ions begin to be formed when evaporation occurs at $40 \mathrm{~V} / \mathrm{nm}$ or below [6]. The evaporation field for tungsten is seen to decrease by $5 \%$ for a $50 \mathrm{~K}$ increase in temperature [7]. Assuming the low temperature evaporation field of tungsten is $57 \mathrm{~V} / \mathrm{nm}$, a temperature rise of $300 \mathrm{~K}$ or above would result in the formation of $2+$ charge states. Since these are not observed, but $4+$ charge states were still observed, we can deduce that the maximum temperature rise was much less than $300 \mathrm{~K}$.

Surface diffusion will be more significant in less refractory materials for a given temperature rise. However, the temperature variation of evaporation field is also greater for less refractory materials, so that a lower temperature rise is required to generate field evaporation. The mass spectrum from intrinsic silicon, Fig. 2, shows that most of the ions are in the $2+$ charge state, with the individual $\mathrm{Si}$ isotopes being resolved and having the expected abundances, to within error. Note that it would have been impossible to analyse this material using voltage pulses. Significant singly charged ions are formed for fields of $20 \mathrm{~V} / \mathrm{nm}$ or below [6]. Assuming the temperature variation for silicon is similar to that of $\mathrm{Fe}$ or $\mathrm{Ni}$ [7], which have similar low temperature evaporation fields and melting temperatures, this would indicate a peak temperature of approximately $150 \mathrm{~K}$. 


\section{References}

[1] A. Cerezo et al., MRS Bulletin 26 (2001) 102.

[2] J.A. Liddle et al., Appl. Phys. Lett. 54 (1989) 1555.

[3] B. Gault et al., to be published in Appl. Phys. Letts.

[4] A. Cerezo et al., J. Microscopy, 141 (1986) 155.

[5] G.L. Kellogg, J. Appl. Phys. 52 (1981) 5320.

[6] G.L. Kellogg, Surf. Sci. 120 (1982) 319.

[7] M. Wada, Surf. Sci. 145 (1984) 451.

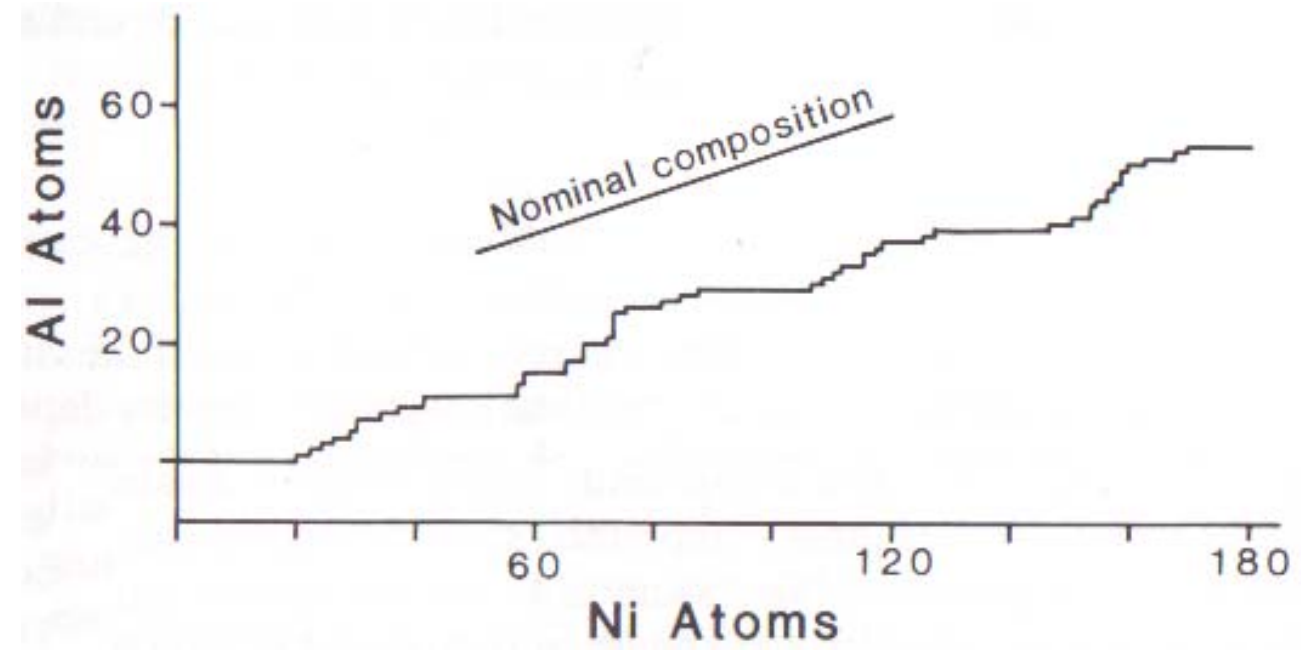

FIG. 1. A ladder diagram obtained from the pulsed laser atom probe analysis along the [100] direction in an ordered Ni3Al alloy. The alternative planes, one of pure $\mathrm{Ni}$ and the other equiatomic $\mathrm{Ni}$ and $\mathrm{Al}$, are clearly resolved [4].

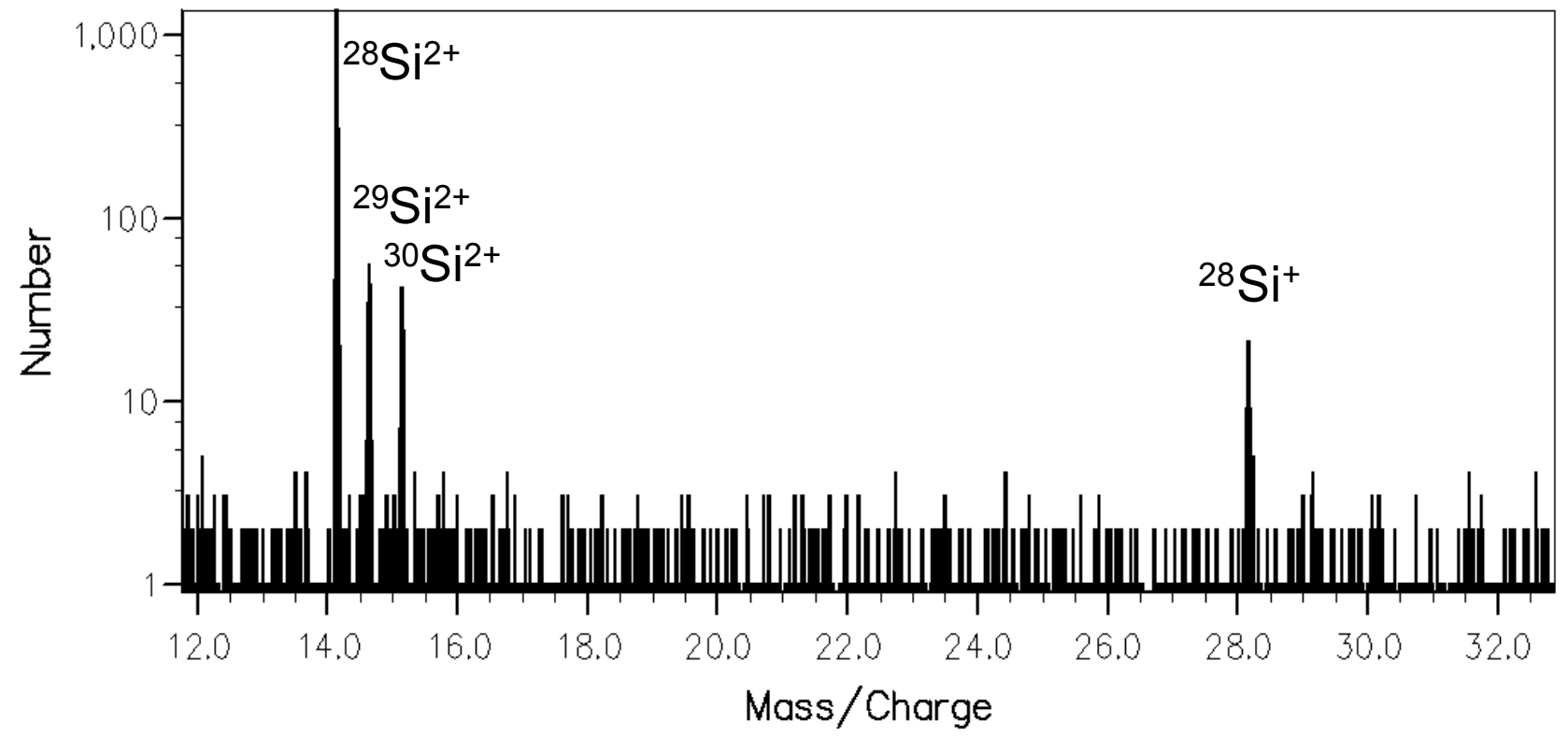

FIG. 2. Spectrum from pulsed laser 3DAP analysis of intrinsic silicon. 